\title{
Efeito da variação da carga orgânica volumétrica natural na produção de biogás a partir de dejeto suíno em diferentes tempos de retenção hidráulica
}

\author{
The effect of organic loading rate's natural variation on the biogas yields \\ from swine manure digestion at different hydraulic retention time
}

\section{Camila Agner D’Aquino** ${ }^{1 *}$, Thiago Carvalho de Mello² $^{\circledR}$, Luis Costa Júnior ${ }^{3}$}

\begin{abstract}
RESUMO
O estudo sobre reatores anaeróbios em laboratório prioriza o controle de determinados parâmetros que são de difícil controle em campo, o que dificulta o processo de scale-up. Entre os parâmetros está a carga orgânica volumétrica (COV). No caso do dejeto suíno, esse parâmetro pode sofrer alterações diárias em função das condições climáticas, das mudanças no manejo, da alimentação dos animais, entre outros. A fim de verificar o impacto dessa variação na produtividade de biogás, foi analisado o comportamento de um reator anaeróbio de mistura completa (CSTR) em dois diferentes tempos de retenção hidráulica (TRHs) - 20 e 30 dias - durante um período total de 180 dias. Os resultados demonstraram que a elevação da COV resulta em piora da eficiência de tratamento, bem como na produção de biogás. O ensaio com TRH de 20 dias obteve uma melhor resposta às alterações de carga orgânica. Palavras-chave: digestão anaeróbia; reator anaeróbio de mistura completa; dejeto suíno; carga orgânica volumétrica.
\end{abstract}

\begin{abstract}
The study of anaerobic digesters at laboratory focuses on the control of certain parameters that are not so easy to control in the field, which makes it difficult to process scale-up. One example is the Organic Loading Rate. To swine manure, this parameter can change daily due to weather conditions, management practices, feeding and others. In order to verify the impact of this fluctuation on biogas yield, the behavior of a CSTR reactor was analyzed in two different Hydraulic Retention Times (2O and 30 days) for a total period of 180 days. The result demonstrated that the increase of OLR result in a decrease of treatment efficiency as well as biogas yields. The study with HRT of 20 days had a better response to changes in organic load.
\end{abstract}

Keywords: anaerobic digestion; continuously stirred tank reactor; swine manure; organic loading rate.

\section{INTRODUÇÃO}

A suinocultura é uma das principais atividades agropecuárias no Brasil. Com a adoção de sistemas de confinamento e de novas tecnologias, há um aumento da concentração de suínos por área e, consequentemente, de produção de dejetos, que contêm altos teores de matéria orgânica, nitrogênio, fósforo, potássio e outros elementos (DUDA; OLIVEIRA, 2009; SOUZA et al., 2008). Quando despejados de forma indiscriminada nos leitos ou aplicados incorretamente ao solo, esse dejeto se torna um passivo ambiental, causando poluição das águas e solos, além de problemas com odor (KARAKASHEV; SCHMIDT; ANGELIDAKI, 2008).

A digestão anaeróbia de dejetos suínos é uma tecnologia de destaque no tratamento de efluentes agroindustriais em razão da sua capacidade de redução do potencial poluidor aliada à produção sustentável de energia por meio do metano e ao abatimento de emissões de gases do efeito estufa (GEE) (KAPARAJU; RINTALA, 2003). Esse processo já é consolidado em muitos países e tem sido visto também como uma fonte de desenvolvimento regional, por intermédio de sua aplicação descentralizada em pequenas propriedades (BATZIAS; SIDIRAS; SPYROU, 2005), o que promove produção local de energia e de um biofertilizante de qualidade.

No entanto, em pequenas propriedades há pouca ou nenhuma disponibilidade de mão de obra qualificada para a operação de reatores complexos. Dessa forma, por conta da simplicidade exigida na operação e da estabilidade, mesmo em situações de mudança brusca do substrato, o reator anaeróbio de mistura completa (CSTR) é indicado

Universidade de São Paulo - São Paulo (SP), Brasil.

Instituto de Tecnologia para o Desenvolvimento - Curitiba (PR), Brasil.

${ }^{3}$ Deutsche Gesellschaft für Internationale Zusammenarbeit (GIZ) - Brasília (DF), Brasil.

*Autor correspondente: camila.daquino@usp.br

Recebido: 11/10/2O13 - Aceito: 15/05/2O18 - Reg. ABES: 124926 
para esse tipo de aplicação (BOE; ANGELIDAKI, 2009). Esse reator tem como principal característica apresentar o mesmo tempo de retenção hidráulica (TRH) e tempo de retenção de sólidos (TRS), situando-se comumente entre 15 e 30 dias (TCHOBANOGLOUS; BURTON; STENSEL, 2003).

Quando pesquisados em laboratório, os parâmetros de estudo são comumente fixados a fim de se observar o comportamento desses reatores de forma controlada. No entanto, quando levados para o campo, é encontrada grande dificuldade de replicação dos resultados obtidos nos estudos laboratoriais em função da variação natural da carga orgânica e de composição do dejeto.

Assim, o objetivo deste trabalho foi verificar os efeitos da variação natural da carga orgânica volumétrica (COV) do dejeto suíno na produção de biogás e na remoção de matéria orgânica em um reator CSTR, em dois diferentes TRHs.

\section{MATERIAIS E MÉTODOS}

\section{Reator e operação}

O fermentador CSTR (Figura 1) utilizado foi projetado e construído como um recipiente cilíndrico de acrílico, com parede dupla para aquecimento. O volume total do reator é de $8 \mathrm{~L}$, o volume operacional é de $6 \mathrm{~L}$ e foi inoculado com 1,2 L de lodo de Estação Anaeróbia de Tratamento de Efluentes coletado na Companhia de Saneamento do Paraná (Sanepar).

Durante 100 dias foi realizado um ensaio com TRH de 30 dias, depois o TRH foi reduzido para 20 dias, tendo esse ensaio durado 76 dias, resultando em um total de 176 dias. Os parâmetros foram mantidos fixos por um período mínimo de três TRHs visando à confiabilidade dos resultados apresentados.

Os outros parâmetros de operação foram utilizados em condições similares. Foi mantida uma faixa de temperatura mesofílica $\left(35,1 \pm 0,4^{\circ} \mathrm{C}\right)$ por meio da recirculação de água na camisa dupla,

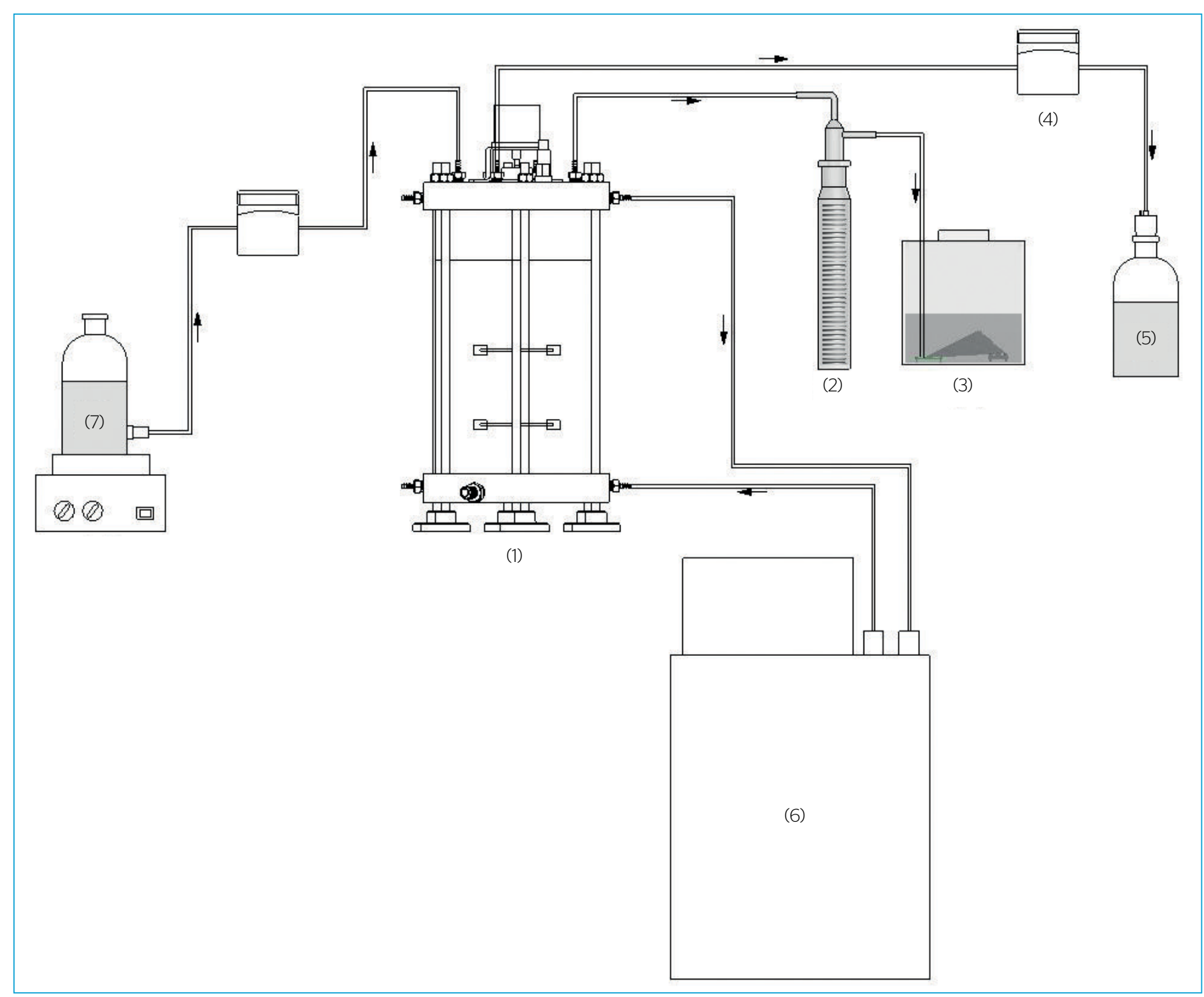

Figura 1 - Fluxograma de operação do reator anaeróbio de mistura completa. 1: reator; 2: lavadora de gás; 3: sistema automático de quantificação de produção de biogás; 4: bomba; 5: efluente; 6 : unidade de aquecimento; 7: afluente. 
aquecida pelo sistema (Figura 1, componente 6). Foram utilizados agitadores do tipo rushton, que promoviam 1 minuto de agitação a aproximadamente 60 RPM seguido por 1 minuto parado (CUBAS et al., 2011).

\section{Métodos analíticos}

A produção de biogás foi medida por intermédio de um dispositivo medidor de volume de gás (Figura 1, componente 3) automatizado e acompanhado por um software de registro de pulsos. O volume foi mensurado por meio do conhecimento do volume da concha. O volume observado foi então normalizado pela Equação 1 (VDI 4630, 2006), descrita a seguir:

$V_{o}^{t r}=V \cdot \frac{(p-p a) \cdot T_{0}}{p_{0} \cdot T}$

Em que:

$V_{o}^{t r}=$ volume do gás seco no estado normal, em $\mathrm{L}_{\mathrm{N}}$;

$\mathrm{V}=$ volume de gás produzido observado no dispositivo, em L;

$\mathrm{p}=$ pressão da fase gasosa no momento da leitura, em hPa;

$\mathrm{p}_{\mathrm{a}}=$ pressão de vapor da água em função da temperatura do ambiente, em hPa;

$\mathrm{T}_{0}=$ temperatura normal, $\mathrm{T}_{0}=273 \mathrm{~K}$;

$\mathrm{p}_{0}=$ pressão normal, $\mathrm{p}_{0}=1.013 \mathrm{hPa}$;

$\mathrm{T}=$ temperatura do gás no interior do reator, em $\mathrm{K}$.

A composição química do biogás produzido foi determinada pela técnica de cromatografia a gás com detecção por ionização em chama (FID) e condutividade térmica (TCD), em cromatógrafo Thermo Finnigan, conforme a NBR 14903 (ABNT, 2008), com adaptações. O pH e a temperatura no interior do reator foram monitorados em tempo real. Para obter a eficiência de tratamento, semanalmente foi analisada a carga orgânica de entrada e de saída do reator por meio da demanda química de oxigênio (DQO) e dos sólidos totais voláteis (STV).

\section{Substrato}

O dejeto suíno bruto foi coletado periodicamente em um colégio agrícola, situado em Castro, Paraná. A coleta foi realizada em frascos de polietileno de $5 \mathrm{~L}$, uma fração foi separada para caracterização no Laboratório de Águas e Efluentes do Instituto de Tecnologia para o Desenvolvimento (Lactec). Enquanto o dejeto não estava sendo utilizado, foi mantido refrigerado a $4^{\circ} \mathrm{C}$, e 24 horas antes de sua utilização ele era retirado e deixado em temperatura ambiente.

\section{RESULTADOS E DISCUSSÃO}

\section{Remoção de matéria orgânica}

O acompanhamento da matéria orgânica de entrada se faz importante a fim de evitar instabilidades, uma vez que os processos fermentativos podem ser alterados por choques de carga orgânica, prejudicando principalmente a metanogênese, pois a população metanogênica é mais sensível a variações de $\mathrm{pH}$, temperatura e carga orgânica (STEINBERG; REGAN, 2011). Neste trabalho, a COV $\left(\mathrm{kgDQO} \cdot \mathrm{m}^{-3} \cdot \mathrm{d}^{-1}\right)$ aplicada foi calculada semanalmente (Figura 2), de acordo com a DQO analisada.

De acordo com Tchobanoglous, Burton e Stensel (2003), em reatores de mistura completa, a COV aplicada pode variar entre 1,0 e $5,0 \mathrm{kgDQO} \cdot \mathrm{m}^{-3} \cdot \mathrm{d}^{-1}$, sem alterar significativamente os processos metabólicos que ocorrem no reator.

No início do ensaio com TRH de 30 dias, a COV ficou abaixo deste valor ideal $\left(0,779 \mathrm{kgDQO} \cdot \mathrm{m}^{-3} \cdot \mathrm{d}^{-1}\right)$, mas depois se manteve dentro dos valores indicados, tendo obtido seu pico no $36^{\circ}$ dia $\left(2,85 \mathrm{kgDQO} \cdot \mathrm{m}^{-3} \cdot \mathrm{d}^{-1}\right)$. Durante o ensaio com TRH de 20 dias, os valores de COV permaneceram dentro do limite sugerido por Tchobanoglous, Burton e Stensel (2003). O pico ocorreu no $3^{\circ}$ dia $\left(2,81 \mathrm{kgDQO} \cdot \mathrm{m}^{-3} \cdot \mathrm{d}^{-1}\right)$ e o menor valor aplicado foi de $1,28 \mathrm{kgDQO} \cdot \mathrm{m}^{-3} \cdot \mathrm{d}^{-1}$

O TRH pode alterar de diferentes formas a eficiência de reatores anaeróbios. Na Figura 3 estão plotados os valores de eficiência de remoção de DQO ao longo dos dois TRHs. Com o TRH de 30 dias, a menor COV aplicada inicialmente teve como resposta alta taxa de remoção de DQO, chegando a 91\% de eficiência.

Com o aumento gradativo da COV, ocorreu decréscimo da eficiência de tratamento, chegando ao mínimo de $48 \%$. Novamente, os valores de COV ficaram mais baixos, resultando em uma eficiência máxima de 75\% de remoção de DQO.

Quando o TRH era de 20 dias, a resposta, em termos de tratamento de matéria orgânica, foi diferente. Quando a COV aumentou, houve também aumento da eficiência de remoção de DQO apresentada, e a máxima COV foi de $2,37 \mathrm{kgDQO} \cdot \mathrm{m}^{-3} \cdot \mathrm{d}^{-1}$, quando a eficiência, também máxima, de $65,7 \%$ foi obtida.

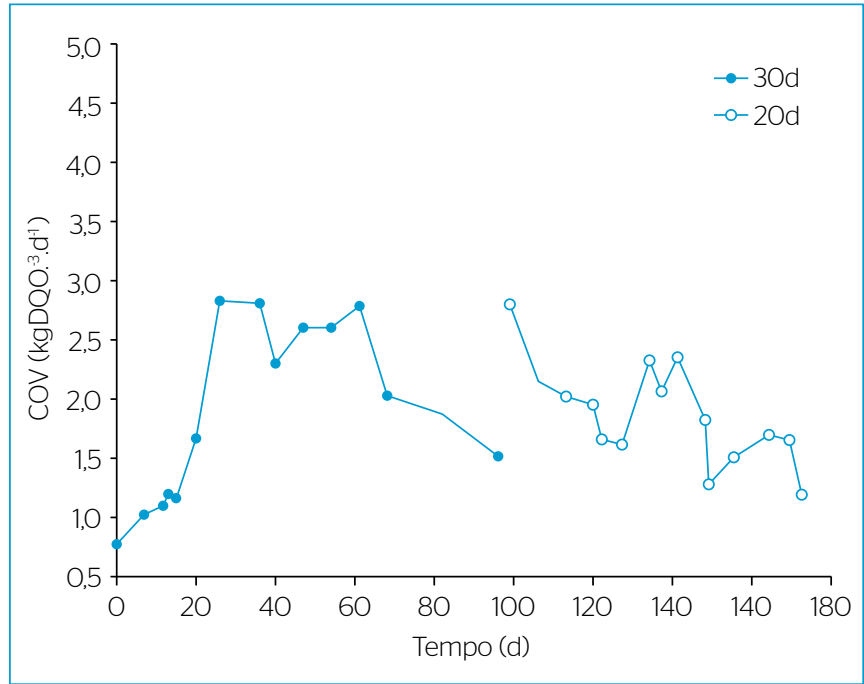

Figura 2 - Variação da carga orgânica volumétrica para cada tempo de retenção hidráulica. 
No TRH de 30 dias, o tempo de resposta, em termos de eficiência de remoção orgânica, foi maior do que quando o TRH era de 20 dias, o que está relacionado ao maior tempo de contato entre a matéria orgânica e o substrato.

Em termos de eficiência de remoção de matéria orgânica, no TRH de 30 dias a média de eficiência foi de $72,9 \pm 14 \%$, enquanto no TRH de 20 dias a média ficou em $52,6 \pm 8 \%$. A diminuição no TRH afetou de forma significativa a eficiência de remoção da matéria orgânica. Com a diminuição da COV, houve também aumento do $\mathrm{pH}$, que passou de, em média, 7,63 (30 dias) para 7,72 (20 dias). Isso pode ser explicado pela diminuição da concentração de ácidos orgânicos voláteis, que impacta negativamente a eficiência do reator devido à influência desses compostos na acidogênese (ABBASI; TAUSEEF; ABBASI, 2012).

\section{Produção de metano}

A produtividade, em termos de biogás, durante os dois períodos encontra-se plotada na Figura 4 e a composição do biogás, em termos da concentração de metano e dióxido de carbono, encontra-se na Tabela 1 . O rendimento de produção de metano em termos de sólidos voláteis e DQO encontra-se na Tabela 2.

No TRH de 30 dias, o máximo de produção foi de $4,0 \mathrm{~L}_{\mathrm{N}}$, no $26^{\circ}$ dia. A média de produção foi de $2,26 \pm 0,75 \mathrm{~L}_{\mathrm{N}} \cdot \mathrm{d}^{-1}$ e o mínimo, de $0,43 \mathrm{~L}_{\mathrm{N}}$, no $11^{\circ}$ dia. No TRH de 20 dias, a média de produção foi de $2,66 \pm 0,63 \mathrm{~L}_{\mathrm{N}} \cdot \mathrm{d}^{-1}$ e o mínimo, de $0,9 \mathrm{~L}_{\mathrm{N}}$ no $46^{\circ}$ dia.

Em termos energéticos, um parâmetro importante é a conversão efetiva da matéria orgânica removida em metano. Sendo que a ineficiência microbiana pode causar a conversão da matéria orgânica em novas células, afetando drasticamente a recuperação do carbono na forma de metano e aumentando, assim, a produção de lodo (CHERNICHARO, 2007).

$\mathrm{C}_{c} \mathrm{H}_{h} \mathrm{O}_{o} \mathrm{~N}_{n} \mathrm{~S}_{s}+\frac{1}{4}[4 c-h-2 o+3 n+2 s] \mathrm{H}_{2} \mathrm{O} \rightarrow \frac{1}{8}[4 c+h-2 o-3 n-2 s] \mathrm{CH}_{4}+$ $\frac{1}{8}[4 c-h+2 o+3 n+2 s] \mathrm{CO}_{2}+n \mathrm{NH}_{3}+s \mathrm{H}_{2} \mathrm{~S}$

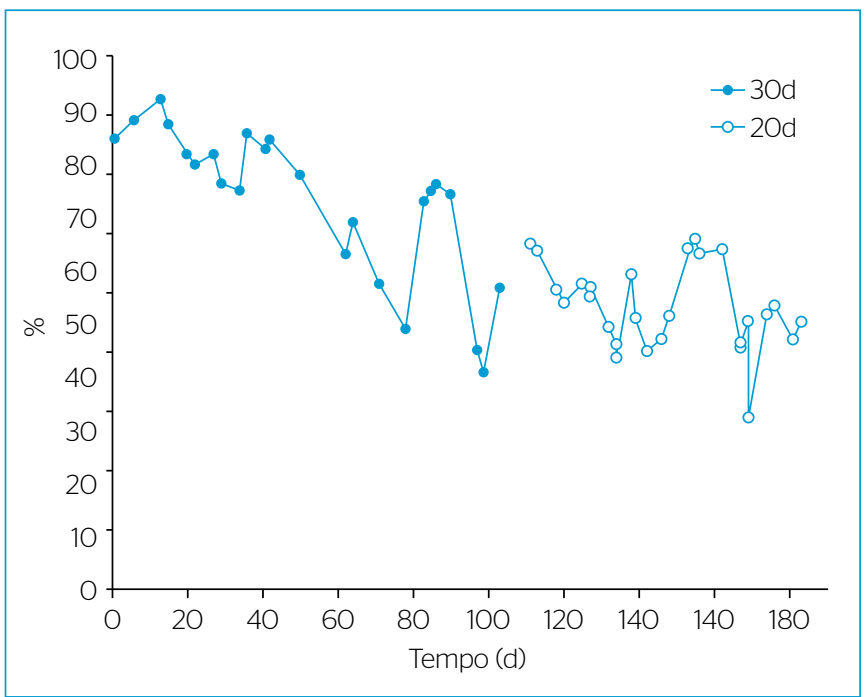

Figura 3 - Eficiência de remoção de demanda química de oxigênio em cada tempo de retenção hidráulica.
Sabe-se que a estimativa da produção de metano a partir da matéria orgânica degradada, em termos de sólidos voláteis, é realizada a partir do conhecimento sobre sua composição e segue a equação de Bushwell (Equação 2), representada a seguir (KHANAL, 2009).

De acordo com estudos realizados Chae et al. (2008), a composição química do dejeto suíno é $\mathrm{C}_{14,25} \mathrm{H}_{28,80} \mathrm{O}_{4,43} \mathrm{NS}_{0,03}$. Portanto, utilizando-se a equação de Bushwell, obtém-se o potencial de produção de metano de $0,72 \mathrm{~L} \mathrm{CH}_{4} \cdot \mathrm{g}^{-1} \mathrm{SV}_{\text {removido }}$

Os resultados apresentados corroboram os reportados por Chae et al. (2008) em condições similares. Em termos de rendimento de metano, não houve diferenças significativas entre os dois TRHs, indicando que, mesmo com a diminuição do TRH, não há dano na capacidade de as bactérias metanogênicas transformarem o acetato em metano. Esse dado pode também ser corroborado pelo teor de metano médio nos dois períodos. Dessa forma, entende-se que a adoção de um TRH menor

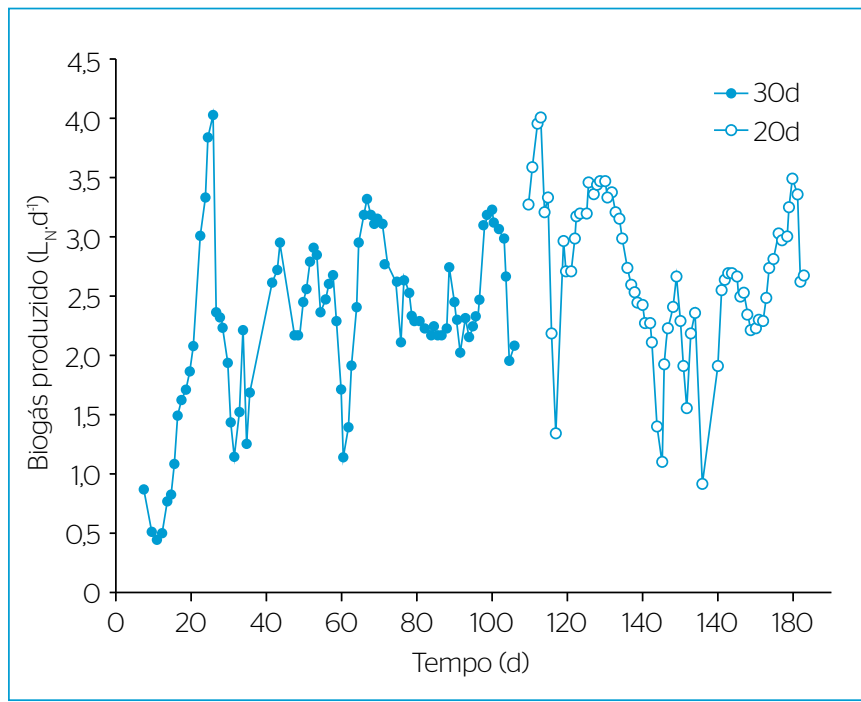

Figura 4 - Produção de biogás em cada tempo de retenção hidráulica.

Tabela 1 - Composição média do biogás nos diferentes tempos de retenção hidráulica.

\begin{tabular}{l|c|c} 
& $\mathrm{CH}_{4}$ & $\mathrm{CO}_{2}$ \\
\hline $30 \mathrm{~d}$ & $70,08 \pm 4,34$ & $19,35 \pm 4,26$ \\
\hline $2 \mathrm{~d}$ & $72,67 \pm 2,13$ & $19,96 \pm 3,60$ \\
\hline
\end{tabular}

Tabela 2 - Rendimento específico de metano para cada tempo de retenção hidráulica avaliado.

\begin{tabular}{|c|c|c|c|}
\hline \multirow{2}{*}{$\operatorname{TRH}\left(d^{-1}\right)$} & \multicolumn{3}{|c|}{ Rendimento em metano } \\
\hline & $\mathrm{L}_{\mathrm{N}} \cdot \mathrm{g}^{-1} \mathrm{SV}_{\text {adicionado }}$ & $\mathrm{L}_{\mathrm{N}} \cdot \mathrm{g}^{-1} \mathrm{SV}_{\text {removido }}$ & $\mathrm{L}_{\mathrm{N}} \cdot \mathrm{g}^{-1} \mathrm{DQO} \mathrm{O}_{\text {removida }}$ \\
\hline 20 & $0,295 \pm 0,064$ & $0,659 \pm 0,197$ & $0,449 \pm 0,145$ \\
\hline 30 & $0,323 \pm 0,190$ & $0,541 \pm 0,343$ & $0,312 \pm 0,180$ \\
\hline
\end{tabular}

TRH: tempo de retenção hidráulica; SV: sólidos voláteis; DQO: demanda química de oigênio. 
é mais vantajoso por reduzir o volume do reator, diminuindo, assim, os custos de implementação e manutenção associados ao processo.

\section{CONCLUSÕES}

Em aplicações do processo de biodigestão em campo para tratamento de dejetos suínos, a COV é um parâmetro largamente alterado de forma natural. Durante o período de TRH de 30 dias, chegou a ser observada alteração em termos de matéria orgânica de mais de $300 \%$. Os choques orgânicos constantes resultaram em quedas imediatas na eficiência de tratamento durante ambos os TRHs, bem como na produção de biogás, principalmente no TRH de 30 dias. Durante o TRH de 20 dias, esses choques de carga orgânica foram menos danosos em termos de produtividade de biogás, mas resultaram em queda mais elevada nos valores de eficiência de tratamento do dejeto. Portanto, em termos energéticos, o TRH menor favoreceu a estabilidade do reator, mesmo com a alteração natural da COV aplicada durante o período de ensaio, enquanto o TRH de 30 dias foi mais eficiente em se manter estável às variações em termos de eficiência de tratamento. A alteração na COV mostrou-se como prejudicial para fins de tratamento por alterar de imediato a eficiência. Para fins energéticos, apesar de ter efeito prejudicial, a alteração na COV é mais bem absorvida durante o processo de digestão anaeróbia, principalmente para o TRH de 20 dias, neste trabalho.

\section{FONTE DE FINANCIAMENTO}

AES Uruguaiana e Programa de Pesquisa e Desenvolvimento (P\&D) da Agência Nacional de Energia Elétrica (ANEEL).

\section{REFERÊNCIAS}

ASSOCIAÇÃO BRASILEIRA DE NORMAS TÉCNICAS (ABNT). (2008) NBR 14903. Gás natural - Determinação da composição química por cromatografia em fase gasosa. São Paulo: ABNT.

ABBASI, T.; TAUSEEF, S.; ABBASI, S. (2012) Anaerobic digestion for global warming control and energy generation - an overview. Renewable and Sustainable Energy Reviews, v. 16, n. 5, p. 3228-3242. https://doi.org/10.1016/j.rser.2012.02.046

BATZIAS, F.A.; SIDIRAS, D.K.; SPYROU, E.K. (2005) Evaluating livestock manures for biogas production: a GIS based method. Renewable Energy, v. 30, n. 8, p. 1161-1176. http://dx.doi.org/10.1016/j. renene.2004.10.001

BOE, K.; ANGELIDAKI, I. (2009) Serial CSTR digester configuration for improving biogas production from manure. Water Research, v. 43, n. 1, p. 166-172. https://doi.org/10.1016/j.watres.2008.09.041

CHAE, K.J.; JANG, A.M.; YIM, S.K.; KIM, I.S. (2008) The effects of digestion temperature and temperature shock on the biogas yields from the mesophilic anaerobic digestion of swine manure. Bioresource Technology, v. 99, n. 1, p. 1-6. https://doi.org/10.1016/j. biortech.2006.11.063

CHERNICHARO, C.A.L. (2007) Reatores Anaeróbios: princípios do tratamento biológico de águas residuárias. $2^{\mathrm{a}}$ ed. Belo Horizonte: Editora UFMG. 380p. v. 5.

CUBAS, S.; FORESTI, E.; RODRIGUES, J.A.D.; RATUSZNEI, S.M.; ZAIAT, M. (2011) Effect of impeller type and stirring frequency on the behavior of an AnSBBR in the treatment of low-strength wastewater. Bioresource Technology, v. 102, n. 2, p. 889-893. http:// dx.doi.org/10.1016/j.biortech.2010.08.128
DUDA, R.M.; OLIVEIRA, R.A. (2009) Reatores anaeróbios operados em batelada sequencial, seguidos de lagoa de polimento, para o tratamento de águas residuárias de suinocultura Parte I: Produção de metano e remoção de DQO e de sólidos suspensos. Engenharia Agrícola, Jaboticabal, v. 29, n. 1, p. 135-147. http://dx.doi.org/10.1590/ s0100-69162009000100014

KAPARAJU, P.L.N.; RINTALA, J.A. (2003) Effects of temperature on post-methanation of digested dairy cow manure in a farm-scale biogas production system. Environmental Technology, v. 24, n. 10, p. 1315-1321. https://doi.org/10.1080/09593330309385674

KARAKASHEV, D.; SCHMIDT, J.E.; ANGELIDAKI, I. (2008) Innovative process scheme for removal of organic matter, phosphorus and nitrogen from pig manure. Water Research, v. 42, n. 15, p. 40834090. https://doi.org/10.1016/j.watres.2008.06.021

KHANAL, S.K. (2009) Anaerobic Biotechnology Production. Singapore: Wiley-Blackwell.

SOUZA, C.F.; CAMPOS, J.A.; SANTOS, C.R.; BRESSAN, W.S.; MOGAMI C.A. (2008) Produção volumétrica de metano: dejeto de suínos. Ciência e Agrotecnologia, Lavras, v. 32, n. 1, p. 219-224. http://dx.doi. org/10.1590/S1413-70542008000100032

TCHOBANOGLOUS, G.; BURTON, F.L.; STENSEL, H.D. (2003) Wastewater Engineering, Treatment and Reuse. 4. ed. Nova York: McGraw-Hill.

STEINBERG, L. M.; REGAN, J. M. (2011) Response of lab-scale methanogenic reactors inoculated from different sources to organic loading rate shocks. Bioresource Technology, v. 102, n. 19, p. 8790-8798. https://doi.org/10.1016/j.biortech.2011.07.017 